\title{
Avvisi a stampa e manoscritti nella Roma del '500
}

\author{
Valeria Sestieri Lee
}

Gli avvisi del '500 costituiscono una fonte d'informazioni di straordinaria importanza per chiunque si interessi alla vita romana di quel secolo. Già da tempo molti studiosi, soprattutto di storia, li hanno consultati; tra questi il Pastor, l'Orbaan, il Delumeau e il D'Onofrio. Ma mentre prima vi si cercavano notizie di personaggi o conferme di eventi storici, da una ventina d'anni si è cominciato a considerarli come espressione di letteratura popolare ed a volgere l'attenzione quindi anche alla loro lingua e al loro stile; inoltre, come fonte di dati socio-antropologici, essi costituiscono una fonte ineguagliata dalle opere letterarie e dai documenti diplomatici a nostra disposizione.

Prima di passare al loro contenuto è opportuno analizzare brevemente la loro natura e gli inizi della loro circolazione sulla scena romana.

Dove e quando incominciarono a circolare in Italia? La risposta dipende da cosa si considera per avviso. Se si accetta la proposta del Barberi che la lettera di Cristoforo Colombo con le prime notizie sulle Indie, stampata a Roma nel 1493 da Stefano Plannck, è un avviso, allora il 1493 è l'anno in cui tali pubblicazioni fanno la loro comparsa, e Roma è la città ove ciò avviene. Nella prima metà del ' 500 ne vengono stampati relativamente pochi e senza alcuna periodicità. Roma rimane tuttavia il centro principale della produzione e diffusione di notizie a stampa; forse perché è uno dei centri urbani più cosmopoliti d'Europa e vi risiede la corte papale, forse perché a Roma vi sono una sessantina di stamperie che stampano tutte avvisi. $\mathrm{Ne}$ stampava pure Antonio Blado, il miglior tipografo romano del '500, dal 1535 impressore camerale con privilegio esclusivo per le pubblicazioni ufficiali della Curia. ${ }^{1}$

Nella seconda metà del XVI secolo la produzione si allarga notevolmente e la tiratura di ogni esemplare sale probabilmente a qualche centinaio di copie, vendute da librai e da ambulanti. Nelle biblioteche romane sono stati ritrovati circa trecentocinquanta avvisi, tre quarti dei quali stampati nella seconda metà del XVI secolo (Bertone 15).

Che notizie diffondono gli avvisi? Continuando ad esaminare quelli a stampa, si osserva che soprattutto i più antichi si limitano generalmente ad un evento straordinario, spesso disastroso: terremoti, inondazioni, eruzioni vulcaniche, apparizioni di comete, incendi, naufragi, fulmini, mostri, pestilenze, carestie, assedi, battaglie, azioni criminali, esecuzioni capitali, i Turchi, feste, miracoli. In seguito, con il passar degli anni, queste pubblicazioni non 
si limitano più ad un solo fatto ed acquistano una, seppur flessibile, periodicità.

La forma letteraria degli avvisi non tiene in alcun conto il livello dei lettori, ossia non mostra la preoccupazione, viva oggigiorno, di indirizzarsi al pubblico tenendosi su uno stesso piano culturale e psicologico. Gli scrittori di avvisi puntavano soprattutto sulla curiosità che un evento straordinario suscitava di per sé, sin dal titolo, ${ }^{2}$ e davano un resoconto degli avvenimenti in una lingua e in uno stile che avrebbero potuto sfoggiare nello scrivere una novella. "Novellanti" sono infatti chiamati coloro che li scrivono, e più spesso "Menanti". L'etimologia di quest'ultima parola è incerta; c'è chi ritiene che venissero chiamati così perché "menavano" l'opinione pubblica. Con più fondatezza si può far risalire il vocabolo al latino medievale "mina" che significa intrigo o mena, e perciò il menante sarebbe un intrigante. ${ }^{3}$

La veste tipografica dell'avviso può essere decisamente definita popolare. Essa è caratterizzata, in toto o in parte, da carta di qualità inferiore, da un formato molto spesso ridotto (in alcuni casi fino ad un ottavo di folio), dallo scarso numero di pagine (a volte soltanto una o due), dall'assenza di spaziatura tra parola e parola e dalla frequenza di errori tipografici. Seppure con eccezioni, il lavoro del tipografo si deteriora progressivamente nel corso della seconda metà del secolo con l'aumento della produzione. ${ }^{4}$

Accanto all'avviso a stampa esiste l'avviso manoscritto. Il Gamrath ritiene che informazioni da "servizio giornalistico" fossero raccolte e redatte in Parione, un rione di Roma, e inviate a più di un "abbonato"; esse riportavano fatti accaduti a Roma e nell'ambito della Curia o dei quali si era sentito parlare in città (49).

Nelle biblioteche e negli archivi di parecchie città italiane esistono raccolte degli avvisi a cui accenna il Gamrath. ${ }^{5}$ La più importante è quella nella Biblioteca Vaticana dei codici Urbinati Latini numerati 1038-1112; i settantacinque volumi coprono eventi che vanno dal 1554 al 1648. I volumi dal numero 1038 al numero 1073 contengono le relazioni dell'ambasciatore urbinate presso la Curia al duca di Urbino. L'ambasciatore non redige le informazioni, ma compra un foglio preparato periodicamente, circa ogni cinque giorni, da un menante che vi ha condensato notizie ricavate da avvisi a stampa e manoscritti e da altre fonti di diversa natura. Non sappiamo se l'ambasciatore urbinate fosse un abbonato o si servisse di un suo menante privato; si pensa senz'altro, però, che gli avvisi manoscritti fossero destinati ad una cerchia ristretta ed anche a singole persone (Bertone 18).

Tutti gli studiosi che hanno fatto ricorso agli avvisi manoscritti concordano nell'attribuire a queste fonti un notevole valore documentario. L'Orbaan (Introduzione) ed il Delumeau (1.50) hanno controllato l'attendibilità dei manoscritti cercando conferma dei fatti in essi riportati in altri documenti ufficiali quali bolle, bandi, decreti, ecc. Gli informatori dei menanti dovevano essere fidati ed altolocati, appartenenti agli alti strati della gerarchia ecclesiastica e amministrativa romana. Gli stretti contatti tra informatori e redattori sono 
evidenziati dalle citazioni di avvisi in rapporti di nunzi e delegati e da stralci di informazioni diplomatiche negli avvisi. Sembra quindi che ci si servisse degli avvisi per preparare il terreno a determinate iniziative e ad operazioni politiche; d'altra parte, attraverso gli avvisi si sondavano le reazioni provocate dalle notizie e si aveva la possibilità di perseguire o abbandonare specifiche linee di condotta politica senza perdita di prestigio. ${ }^{6}$

I menanti dimostrano una buona dose di acume e di cautela nell'analizzare le notizie ricevute. L'episodio dell'Invincibile Armata ne è un chiaro esempio. La Curia dava come vincitori gli Spagnoli e solo alla lunga trapelò a Roma la notizia della loro disfatta. Gli avvisi riportavano le voci che circolavano, ma sottolineando che non si aveva conferma alcuna dei fatti; un breve stralcio da un avviso fa luce sul modo di operare dei menanti e sulla esclusività delle informazioni da loro procurate: "Ancorché questi spagnoli tenghino secreti li loro avisi, nondimeno si sa, che con l'ultime tre hanno raguaglio come l'armata Cattolica stava alla vela senza dirsi a qual volta ..."?

La scelta delle notizie riportate qui di seguito ${ }^{8}$ è stata motivata dalla loro natura; curiosa, orrorosa, o comunque interessante per lo stile, per la vivacità e per l'umorismo con cui tali notizie vengono riferite. Il primo stralcio, di una certa lunghezza, darà un'idea della varietà di informazioni che ciascun avviso conteneva.

Il Gran Duca di Firenze ha mandato a donare 70 piatti d'argento al Cardinale di Cosenza.

Non è vero che li prigioni per conto il già Cardinale Rambuglietto habbiano confessato nulla di havere avelenato Sua Signoria Illustrissima come pretendevano i parenti del Cardinale essendosi trovato veramente in cassa quella quantità di elleboro bianco che si pretendeva essere stato posto in un servitiale fatto al Cardinale, di cui ordine si è scoperto che fosse stato preso detto elleboro per sanare, $\mathrm{o}$ fare certa medicina ad un cavallo.

L'Ambasciatore di Spagna si vede da alcuni giorni in qua tratta molto alle strette con alcuni Cardinali della Corte più spesso del solito, come fa con Rusticucci ancorché Sua Signoria Illustrissima si trovi in letto con podagra, et febbre, desiderando ognuno che '1 negotiato di cui Ambasciatore sia per conto dell'Impresa d'Inghilterra, et dicendosi che Sua Signoria sborserà (come s'habbia aviso dell'arrivo dell'Armata di Spagna nella Britannia) li 800 scudi promessi fra dui mesi per questa impresa di sua parte a perpetua gloria di Sua Benedizione.

È morto il signor Bernardo Aldobrandino fratello del Cardinale, che haveva moglie; (È vero, et Sua Signoria Illustrissima la sente estraordinariamente). ${ }^{9}$

Questi avvisi redatti periodicamente permettono anche di seguire gli sviluppi di un avvenimento. La misteriosa vicenda della morte del Cardinale de 
Rambouillet non si esaurisce con quanto appena riportato. A distanza di otto giorni leggiamo:

I Carcerati servitori del già Cardinale Rambuglietto si crede, che presto faranno di loro brutta mostra per la città, per lo elleboro bianco che lo spetiale confessò da principio di havere posto nel servitiale fatto al Padrone havendo forsi questi meschini per i tormenti deposto quello, che i medici disputano tra loro se sia bastata certa poca quantità di elleboro bianco ad ammazzare Sua Signoria Illustrissima di che Nostro Signore nella signatura passata dicono facesse parola dicendo che con suo grande dispiacere haveva sentita l'infedeltà di quei servitori.

Quattro mesi dopo:

Furono rilasciati li servitori del già Cardinale Rambuglietto, et data loro la casa per carcere con buona sicurtà finché siano finiti certi atti, et poi si liberaranno affatto dallo spetiale in poi, che sarà apiccato, o mandato in galera, non aspettandosi altro per l'essecutione, che la voluntà del Papa, et il ritorno di Sua Santità essendosi in questa causa conosciuta veramente la innocenza di questi poveri Italiani, che hanno havuti tanti tormenti a torto per querele lor date dalli parenti il morto Cardinale il cui fratello è hora cercato dalla corte per 2600 scudi imprestatigli dalli sudetti Italiani.

E nel seguente invio di notizie:

Si va cercando il fratello del Cardinale Rambuglietto per metterlo pregione per onere delli 2600 scudi havuti imprestito dalli servitori d'esso Cardinale, cavati di pregione, et datoli la casa per carcere, come innocentissimi eccetto lo spetiale.

Che giustizia venisse fatta è evidente, non solo da molti fatti di cronaca riportati negli avvisi, ma anche da tanti bandi, decreti e lettere ufficiali che negli stessi anni proclamavano al popolo nuove leggi e regolamenti diretti ad ordinare e controllare la vita dell'Urbe. Nel confronto tra bando e avviso si notano alcuni fatti degni di nota. Mentre la lingua e lo stile del bando sono ufficiali e altisonanti, lo stile dell'avviso è di frequente economico, quasi telegrafico. Si rifugge dall'elaborazione: "et quest'anno si può dire che quasi più son state le teste in Ponte ch'i Meloni in Banchi". ${ }^{10} \mathrm{Si}$ usa spesso la parola precisa, scarna; quando la frase è colorita e descrittiva vi si avverte una partecipazione sentimentale, quasi una presa di posizione dello scrittore, come nei già citati "havendo forsi questi meschini per i tormenti deposto quello" o "essendosi in questa causa conosciuta veramente la innocenza di questi poveri Italiani”, dove si rileva poco meno che una nota di gallofobia. Inoltre le proclamazioni dei bandi restano distaccate e distanti dagli individui a cui sono indirizzate; che si tratti di esseri umani i quali, per la maggioranza, campano una vita grama, ce lo svelano gli avvisi.

Nel luglio del 1586 uscì il bando "de non portare Archibugi in Campagna"; proclamava:

L'illustre e Reverendissimo Monsignor Mariano Perbenedetti, Vescovo di Martorano, di quest'alma Città di Roma e suo distretto general Governatore e Vicecamerlengo, 
considerando che con havere concesso tante licenze di portar Archibugi in Campagna essersi molto trascorso e si vede che ogn'uno indifferentemente portarli in modo che causano disturbo pericolo e danno, onde per degni rispetti Sua Signoria Reverendissima per ordine di Nostro Signore Revoca tutte le licenze . . . Però ogn'uno se guardi di non contravenire, perché se procederà con ogni rigore irremissibilmente.

Quasi della stessa data è un altro avviso:

È fuori un bando con prohibitione a metitori di portar armi in campagna, con tutto, che di questi meschini l'anno passato verso la marina, i Corsari per trovarli disarmati per un bando simile ne pigliassero in buon numero.

Le condizioni precarie e brutali della vita umana del tempo ci vengono messe sotto gli occhi quasi ad ogni passo:

Hieri di notte furono mandati in galera 40 condannati; et delli Turchi mandati qua dal legato Canano, 8 il Papa ne ha donati a Maltesi, 8 alli cavalieri di Santo Stefano, et altri tanti a Genovesi, et se quei 14 giocatori presi nella Franchigia de Medici non sono aiutati da qualche santo, andaranno ancor essi ad triremes, et di quella Donna carcerata al sant'Officio per haver in publico affermato, che Nostro Signore morirà (il che a Dio non piaccia mai) per tutto certo ottobre prossimo, non si sa che sarà di lei.

Un certo Giovanni Fiamengo Astrologo, che non ha saputo così predire il suo danno, come quello degli altri, stette un pezzo l'altro giorno in Berlina.

Stamane è stata tagliata la mano al luogo del delitto a quello che uccise in Banchi un pezzo fa un certo Paolo Pietro da Toscanella di cui si scrisse, et poi impiccato e squartato et seco sono stati giustiziati tre ladri molto celebri.

Gli indemoniati che fanno tanto strepito e resistenza per non entrare in quelle caverne rimote, che si scoprono sotto Monte Magnanapoli per trovare quei Corpi santi scritti, danno segno evidente della santità di quel luogo e che là sotto stiano cose sacre. ${ }^{11}$

Viene notato che dal giorno di Pasqua in qua sono morti in questa Città 19 medici, con gusto grande di quelli, che non sono della lor professione (et è verissimo). ${ }^{12}$

È qua venuto uno di Spagna, che testifica contro quel Pietro Antonio già sostituto del Contarello la cui causa non per altro ha dormito fin adesso, che per questo, et per altri testimoni, che si aspettavano (contro lui non hanno fin qui altro che sofisticherie).

Seguendo i costumi a cui i Romani erano abituati da molto tempo, durante il Carnevale veniva data al popolino la possibilità di dimenticare le strettezze della vita di ogni giorno.

Sabbato fu dato principio qua alle commedie, ma ben presto prohibite, dicendo Nostro Signore quando ne hebbe notizia, che molto meglio farebbero quelli che ne sono 
curiosi, di andare alle prediche. Si tiene però, che si permetteranno senza fallo le maschere per spasso della Plebe sotto i soliti bandi.

I Comici Desiosi hanno ottenuto licenza con porgere certa elemosina a luoghi pij di recitare le loro commedie in Roma, et hoggi vi hanno dato principio con molto gusto del Popolo pagando ciascuno che vuol vederle una certa miseria. Né possono (per levare i scandali) intervenirvi donne, et Huomini con armi di qualsivoglia sorte.

Si è dato qua principio fin da lunedì al correre dei pallij secondo il consueto, et ad alcuni che hanno havuto ardire di attraversare la strada del Corso mentre si correva, sono stati dati tre tratti di corda secondo $\mathrm{i}$ bandi, et poi carcerati, et oltre a questi spassi, et delle maschere, vi è anco quello delle commedie, et tragedie molto belle che si recitano giornalmente da questi Comici Desiosi con gran concorso, pagandosi ogni volta da ciascuno, che vuol udire la commedia un giulio, et due della Tragedia, et vanno anco i medesimi Comici la sera per le case dei Signori particolari, ove sono chiamati a recitarle per una decina di scudi per volta, ... et così passiamo il Carnevale allegramente.

Vivo appare il contrasto tra la condizione della plebe e quella dei privilegiati dalla sorte. Di una di costoro ci vien detto:

Fu provvisto dalla clementia di Nostro Signore al vivere quotidiano, et a gli altri bisogni della Gentildonna Greca fuggita d'Algieri con assegnamento di 30 scudi al mese, et la casa pagata per 80 scudi l'anno, lasciando al servitio di Lei due di quelle schiavette, che condusse seco d'Algeri, et molti Cardinali porgeranno anco le mani loro adiutrici in sì santa opra separatamente da Nostro Signore si come ha fatto $\mathrm{Pa}$ leotto, che gli ha mandato a donare una croce piena di Rcliquie con 50 scudi d'oro, et alcune galanterie Napoletanc.

\section{Ancora:}

Martedì sera il signor Ciriaco Mattei con l'occasione delle nozze del signor Giovanni Battista suo figliolo fece un sontuosissimo banchetto a 14 Cardinali, che mangiaro in disparte col Cardinale Mattei suo fratello, et a 60 Dame ... di questa Città con gran numero di Prelati, ct signori, essendo state servite 6 gran tavole tutte ad un tempo con sì bell'ordine, silentio, quiete, et splendore, che maggiore non si poteva desiderare. Fu danzato prima, et da poi della cena, et recitata appresso una commedia intitolata de quattro simili molto bella.

Il Cardinale Albano, che sta in letto con inappetenza, et male in una gamba, ha donato un corsiero di 500 scudi al Cardinale Alessandro, che glielo haveva dimandato in vendita, il quale Alessandro dopo avere patito un pezzo di renella ha orinata una grossa pietra.

Un passatempo diffusissimo che accomunava tutte le classi sociali era quello delle scommesse. Oltre a vendere sogni a buon mercato esse costituivano un'ottima rendita per il governo. I banchi per le scommesse venivano 
dati in appalto a dei sensali e, durante il regno di Sisto $\mathrm{V}$, vi sono bandi e nuovi bandi intesi a controllare le scommesse. Se ne parla anche negli avvisi:

Il Papa si piglia gusto incredibile di vedere le liste delle scommesse, che si fanno in Banchi sopra i purpurandi et sopra altri casi futuri.

S'accende maggiormente l'opinione fra tutti di Roma, che il Papa andarà a quel viaggio, che s'è scritto, et in banchi le scommesse sono a 20 giuli per cento, che per tutto settembre sarà partito, ancorché molte ragioni obstino a questo, se bene, et solamente si discute sopra a tal andata.

Una tale sicura fonte di entrate veniva osservata con interesse anche da altri governanti. Infatti:

Si tratta di erigere un Monte sopra le scommesse, ... et come ciò si eseguisca in Roma anco il Gran Duca le permetterà nel suo stato.

Ma nel 1590, non potendo presumibilmente imporre un controllo efficace sui sensali e le loro transazioni, il Papa emette il "Bando prohibitorio delle scommesse":

Essendosi per esperienza visto le molte liti, et inconvenienti che sono nati dal far le scommesse in la maniera che fin al presente si sono fatte . . , col disturbo publico d'ogni stato, e qualità di persone . . . , per tenore del presente pubblico bando, di ordine espresso di sua Beatitudine, e per l'autorità del nostro Offitio, ... prohibemo, ordinamo e espressamente comandamo a qualunque persona di qual si voglia stato, ... che ... non ardischi, presumi per sé o per altri ... fare, o far fare alcuna sorte di scommessa sopra a suggetti a promotioni de Cardinali, o altre concessioni di dignità o Offitio da farsi dalla Santità di Nostro Signore, se sopra maschio o femina, sotto pena a chi contraverrà ... .

Le pene erano pecuniarie, corporali e comportavano fino a cinque anni di esilio.

Gli avvisi ci danno anche numerose informazioni sull'attività del Papa e della Curia, dalle manovre politiche ai provvedimenti presi per l'andamento economico e sociale dello Stato, dalle numerose imprese edilizie intraprese per il rinnovamento urbano e sociale di Roma agli interessi culturali di Sisto. Si viene a sapere che il pontefice progettò di riattare il Colosseo per istallarvi botteghe e magazzini per l'Arte della Lana ed altre Arti, ed alcuni artigiani vi aprirono bottega; che quattro bellissime colonne di serpentino furono rimosse dalla chiesa di San Pietro in Vincoli per essere riusate altrove e che la popolazione reagì negativamente perché furono sostituite con altre nemmeno di travertino, ma di mattoni; che Nostro Signore richiese ad una Congregazione di Cardinali di trovare nuove fonti di denaro "senza aggravio dei suoi Popoli" dato che "li 4 milioni d'oro che ha Sua Beatitudine in Castello sono una insalata rispetto al suo urgentissimo bisogno". Sisto visitava regolarmente tutte le fabbriche iniziate, rinnovate o restaurate per suo volere "salendo per tutte 
quelle volte, et stanze con dispositione giovanile (Dio lodato)"; si interessava di far spostare la libreria del Vaticano in locali meno umidi e si premurava di aumentare le collezioni della libreria; dava licenza di far stampare una "Bibbia in lingua Siriaca, Caldea, Arabica, Persiana et Ethiopica per mandarne in quelle Regioni a quei Popoli, che la desiderano per la cathechizzazione loro", come pure considerava la proposta di un architetto di usare molti obelischi fatti erigere in varie piazze romane come "Herologij", "Cosa in vero molto bella".

Gli avvisi manoscritti danno un quadro complesso e dettagliato della vita romana e ben vi si accompagna la vivacità e l'interesse con cui son redatte le notizie riportate. Il menante non è né un burocrate né un diplomatico. Ma cosa lo spingeva a scrivere? L'attrazione di divulgare notizie, un'inclinazione letteraria o la necessità di sbarcare il lunario? Vero è che non conduceva un'esistenza facile. Le sue prestazioni erano necessarie, forse anche apprezzate dai suoi clienti e spesso ben pagate, ma il suo era mestiere difficile, persino rischioso.

Da quando nel 1565 gli avvisi periodici a stampa, ormai meglio organizzati, vanno sotto il nome di "Avvisi delli avvenimenti del mondo", i pontefici si accaniscono con un susseguirsi di bandi contro "l'Arte nuova". Così è definita la menanteria nella Costituzione del primo settembre 1572 emanata da Gregorio XIIJ. Sisto V si scagliò con straordinaria acredine contro i menanti nel "Bando contra li calunniatori e detrattori della fama et honor d'altri", dell'11 novembre 1586 , in cui tra l'altro è scritto:

... si ritrovano alcuni di tanto mala natura scelerati calunniosi e detrattori che senza timor di Dio et della giustizia . . hanno pigliato l'occasione, per esercitare le loro lingue pestifere, de scrivere lettere d'avisi ... empiendo le carte de bugie et calunnie, infamando e detrahendo all'honore et reputation del prossimo ...

Le pene stabilite dal bando erano: condanna a morte, confisca dei beni ed infamia perpetua. Alle parole seguirono i fatti. Un avviso riporta:

Ier sera fu degradato in San Salvatore in Lauro quel don Annibale Cappello, et questa mattina è stato condotto al luogo solito della giustizia in Ponte. Dove prima gli è stata mozzata una mano, tagliato la lingua et impiccato con tale discriptione: Per menante falso, detrattore per molti anni delli gradi di persone d'ogni sorte, . . . et per haver scritto avvisi ai prencipi heretici. ${ }^{13}$

La licenza per gli avvisi fu imposta con un bando del settembre 1602. Nel marzo del 1621 in un "Bando generale concernente il Governo di Roma", pur ribadendo la necessità della licenza per i menanti, si distingueva tra questi e i libellisti. Si riconosceva cioè la legittimità della diffusione di notizie e di chi l'aveva scelta per mestiere.

University of Calgary 


\section{NOTE}

1 Per notizie sulle stamperie romane vedi Ascarelli e Barberi.

2 Ad esempio, in Bulgarelli 131-4 è riprodotto un avviso del 1534 dal titolo "Letera de la nobil cipta: novamente ritrovata alle Indie con li costumi e modi del suo Re e soi populi: li modi del suo adorare con la bella usanza de le donne loro: e de le dua persone ermafrodite donate da quel Re al Capitano de l'armata".

3 Nel Bando di Sisto V dell'11 novembre 1586 i menanti vengono chiamati scellerati, calunniatori e detrattori della fama e onore altrui. Vedi D'Onofrio 541 e Delumeau 1.30.

4 Per la veste tipografica ed anche per la forma letteraria degli avvisi vedi Petrucci Nardelli 262.

5 Così nelle Biblioteche Nazionali di Roma, di Napoli e di Firenze, alla Marciana di Venezia ed in archivi e biblioteche di altre città italiane come Lucca, Mantova, Modena, Torino. Per queste ed altre notizie sugli avvisi e gazzette del XVI e XVII secolo vedi anche Stumpo, Introduzione.

6 Per il modo di operare dei menanti e le loro fonti d'informazione cf. Gamrath 50.

7 Avviso ms. del 12-5-1590 nel codice Urbinate Latino 1058, f. 235 r. Nel riportare gli avvisi si è mantenuta la forma dell'originale eliminando, però, le abbreviazioni.

8 Le notizie sono tratte dai volumi 1053-1058 dei codici Urbinati Latini che coprono gli anni 1585-1590, ossia gli anni del pontificato di Sisto V. Sono state riportate senza osservare un ordine cronologico.

9 Le parole in parentesi alla fine sono di un'altra mano.

10 "Ponte" è ponte Sant'Angelo ad un capo del quale venivano eseguite buona parte delle sentenze capitali. "Banchi” è una strada vicina a ponte Sant'Angelo dove erano situati molti banchi per il cambio, per il prestito di denaro e per le scommesse.

11 Qui e altrove la parola "scritto" (al maschile, femminile, singolare e plurale) indica che persone o fatti sono già stati menzionati precedentemente.

12 L'avviso è del 22 luglio.

13 Va qui chiarito che la severità della condanna che colpì Annibale Cappello era dovuta soprattutto al fatto di aver servito degli eretici ed aver scritto anche libelli pornografici; egli non era un comune menante.

\section{OPERE CITATE}

Ancel, René. "Étude critique sur quelques recueils d'avvisi". Melanges d'archeólogie et d'histoire 28 (1908): 115-139.

Ascarelli, Fernanda. Le cinquecentine romane. Censimento delle edizioni romane del XVI secolo possedute dalle biblioteche di Roma. Milano: Etimar, 1972.

La tipografia cinquecentina italiana. Firenze: Sansoni 1953.

Barberi, Francesco. "Gli avvisi a stampa nella Roma del Cinquecento". Strenna dei Romanisti (1955): 277-281.

“Libri e stampatori nella Roma dei Papi”. Studi Romani 13 (1965): 433-456.

Bertone, A. et al. Il Giornalismo romano delle origini (sec. XVI-XVII), catalogo della mostra. Roma: Biblioteca Nazionale Centrale, 1979.

Bulgarelli, Tullio. Gli Avvisi a stampa in Roma nel Cinquecento. Bibliografia-Antologia. Roma: Istituto di Studi Romani, 1967.

Delumeau, Jean. Vie économique et sociale de Rome dans la seconde moitié du XVI siècle. Bibl. des Écoles fr. d’Athènes et de Rome, 184. Paris: De Boccaro, 1957-59.

D’Onofrio, Cesare. "Gli avvisi di Roma dal 1554 al 1605 conservati in biblioteche ed archivi romani”. Studi Romani 10 (1962): 529-548.

Gamrath, Helge. Roma Sancta Renovata. Roma: L’Erma di Bretschneider, 1987.

Orbaan, Jacob F. "La Roma di Sisto V negli Avvisi". Archivio della Società Romana di Storia Patria 33 (1910): 277-312. 
Pastor, Ludwig von. Storia dei Papi. Vol. X. Roma: Desclée e C., 1928.

Petrucci, Armando, ed. Scritura e popolo nella Roma Barocca 1585-1721. Roma: Quasar, 1982.

Petrucci Nardelli, Franca. "Calamità e paure nella stampa popolare romana e laziale (15851721)". Archivio della Società Romana di Storia Patria 105 (1982).

Stumpo, Enrico. La Gazzetta de l'Anno 1588. Firenze: Giunti, 1988. 